\title{
Emotionsloser Zeuge einer Tragödie ${ }^{1}$
}

\section{Unemotional Witness to a Tragedy}

\author{
Autor \\ H.-D. Göring \\ Institut \\ Tumorzentrum Anhalt am Städtischen Klinikum Dessau e. V. \\ (Vorsitzender: Prof. Dr. med. H.-D. Göring)
}

Bibliografie

DOI 10.1055/s-0028-1119703

Online-Publikation: 13.3. 2009

Akt Dermatol 2009; 35:

351-353 @ Georg Thieme

Verlag KG Stuttgart · New York ISSN 0340-2541

Korrespondenzadresse

Prof. Dr. med.

Hans-Dieter Göring

Tumorzentrum Anhalt

am Städtischen Klinikum

Dessau e. V.

Auenweg 38

06847 Dessau-Roßlau

tzd@klinikum-dessau.de

\section{Zusammenfassung}

$\nabla$

Dr. med. Gottfried Benn hat im Oktober 1915 als Beobachter am Prozess gegen die britische Krankenschwester Edith Cavell vor einem deutschen Kriegsgericht im besetzten Brüssel teilgenommen. Wegen „Zuführung von Mannschaften an den Feind“ wurde Edith Cavell zum Tode verurteilt. Benn war als Militärarzt zur anschließenden Erschießung der Delinquentin am 12.10.1915

\section{Einleitung \\ $\nabla$}

Dr. med. Gottfried Benn, angehender Dermatologe und später einer der bedeutendsten deutschen Lyriker des 20. Jahrhunderts, fand im Ersten Weltkrieg nach vergeblichen Versuchen, im bürgerlichen Leben Fuß zu fassen, durch die Ausnahmesituation Krieg paradoxerweise zu sich selbst zurück [1]. Die Erstürmung von Antwerpen im Herbst 1914, an der er als Militärarzt teilnahm, blieb sein einziger Fronteinsatz im Ersten Weltkrieg. Der opferreiche Stellungskrieg an der Westfront war weit weg, als er im Oktober 1914 Leiter eines Prostituiertenkrankenhauses im von kaiserlich-deutschen Truppen besetzten, aber nicht umkämpften Brüssel wurde [1] ( $\bullet$ Abb. 1). Eine Konzentration zahlreicher deutscher Soldaten einerseits und die häufig aus wirtschaftlicher Not geborene Prostitution belgischer Fabrikarbeiterinnen andererseits sowie der kriegsbedingte Verfall von Moral und Sitten auf allen Seiten führten zu einem rasanten Anstieg venerischer Erkrankungen, denen sich Benn als Arzt gegenübersah [2]. Trotzdem fand er genügend Zeit, um schriftstellerisch zu arbeiten. Als Lyriker war er bereits 1912 mit dem schmalen Gedichtband „Morgue und andere Gedichte“ quasi über Nacht bekannt

${ }^{1}$ Herrn Prof. Dr. med. Wolfgang Chr. Marsch zum 60. Geburtstag gewidmet. kommandiert worden. Erst dreizehn Jahre später hat er am 22.2.1928 in einem Artikel für das „8 Uhr-Abendblatt der Nationalzeitung“ darüber berichtet. Gottfried Benn schildert darin mit einer, zumal für einen Arzt, kalten Distanziertheit dieses Ereignis. Das tragische Schicksal von Edith Cavell ist weltweit in Büchern und Zeitschriften beschrieben und verfilmt worden. Es weckt in Großbritannien bis zum heutigen Tage Anteilnahme.

geworden. 1913 folgte die Gedichtsammlung „Söhne“, die er Else Lasker-Schüler gewidmet hatte. In Brüssel traten nun auch Prosa und Dramatik in sein Werk, die literarischen Figuren Rönne und Pameelen nahmen Gestalt an. Glückliche Jahre wird er später diese Zeit nennen [1].

Im Unterschied zu einer Reihe anderer Schriftsteller, die ihre Erlebnisse im Ersten Weltkrieg in später berühmt gewordenen Romanen gestalteten (u.a. Erich Maria Remarque: „Im Westen nichts Neues“, Ernst Jünger: „In Stahlgewittern“, Ludwig Renn: „Krieg“, Arnold Zweig: „Der Streit um den Sergeanten Grischa“), haben Krieg und revolutionäre Nachkriegswirren kaum direkte Spuren in den Essays und der Lyrik Gottfried Benns hinterlassen [2]. Zwei Ereignisse aus dem Jahre 1915 haben sich aber unauslöschlich in Benns Gedächtnis eingegraben. Zum einen war das sein Zusammentreffen mit seinem von den Kämpfen an der Westfront schwer gezeichneten jüngeren Bruder Siegfried in Brügge. Kurze Zeit später fiel der 22-Jährige an der Ostfront in Galizien. Diesen tragischen Tod hielt Benn 1933 in dem Gedicht „In memoriam Höhe 317“ fest [2]. Das andere Ereignis war die Erschießung der britischen Krankenschwester Edith Cavell am 12. Oktober 1915 in Brüssel, an der Benn als abkommandierter Militärarzt teilnahm. Erst 13 Jahre später, am 22. Februar 1928, berichtete Benn über den Kriegsgerichtsprozess gegen Edith Ca- 


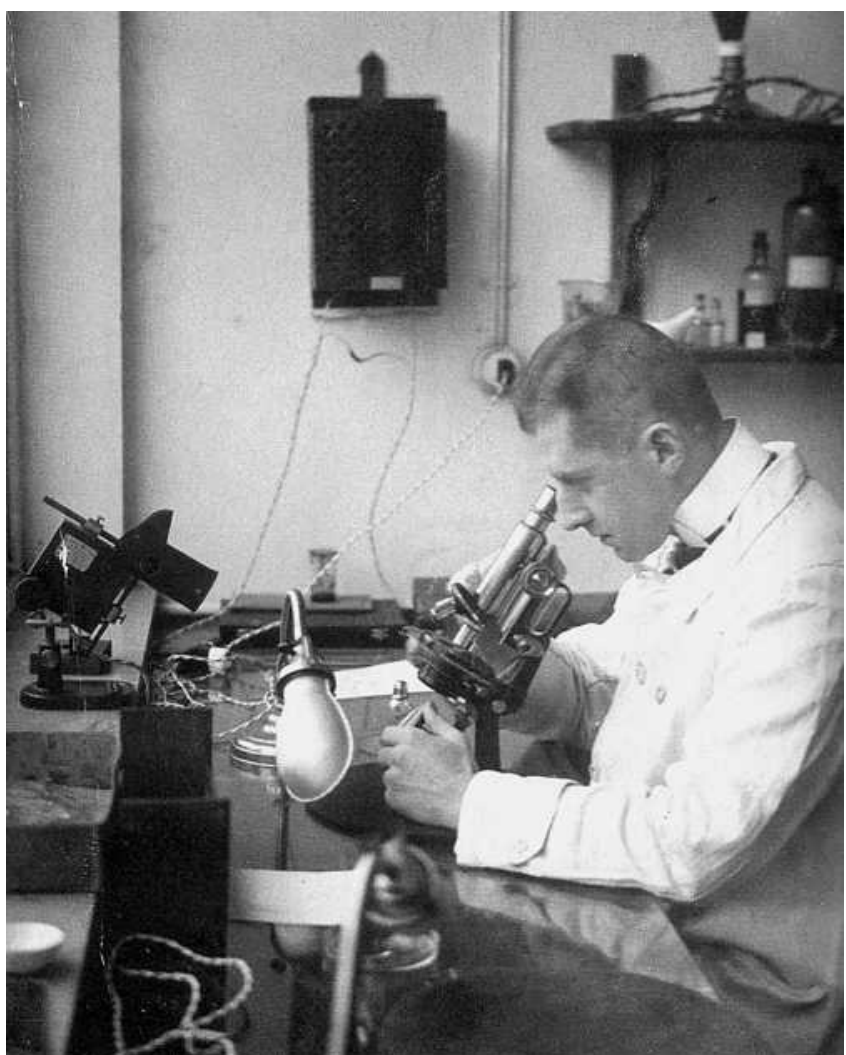

Abb. 1 Dr. med. Gottfried Benn als Militärarzt in Brüssel (aus [4]).

vell und den belgischen Ingenieur Boog (Baucq) und die Hinrichtung der beiden Delinquenten im „8 Uhr-Abendblatt der National-Zeitung“ [3] ( Abb. 2). Edith Cavell, eine britische Krankenschwester und Leiterin einer Schwesternschule, lebte schon viele Jahre vor Ausbruch des Ersten Weltkrieges in Brüssel. Zu Beginn des Ersten Weltkrieges hatten sie und ihre Helfer, zu denen auch Boog gehörte, unter dem Deckmantel einer Einrichtung des Roten Kreuzes mehrere Hundert wehrfähige Belgier und französische Soldaten auf Schleichwegen über die belgisch-holländische Grenze zum Kriegseinsatz gegen das deutsche Heer geführt $[1,2]$. Nach ihrer Festnahme waren die 50-jährige Edith Cavell und der etwa 40-jährige Boog von einem deutschen Kriegsgericht wegen „Zuführung von Mannschaften an den Feind“ zum Tode verurteilt worden, obwohl zum Zeitpunkt der Verurteilung auf dieses Delikt nicht die Todesstrafe stand (Wandt [2]). Juristisch war außerdem nicht klar, ob Spionage vorlag. Daher ist eine Gleichsetzung des Falles von Edith Cavell mit dem der 2 Jahre später von einem französischen Kriegsgericht zum Tode verurteilten angeblichen deutschen Spionin Mata Hari, wie sie der Benn-Biograf Walter Lennig vornahm [4], falsch. Gegen Spionage im Fall Edith Cavell sprach auch die Tatsache, dass der Prozess gegen sie und Boog öffentlich war und beide Angeklagten belgische Verteidiger hatten [2]. Was Gottfried Benn nicht wusste oder nicht zu wissen vorgab, war, dass hinter den Kulissen diplomatisch um das Leben von Edith Cavell gerungen wurde. Durch die Zögerlichkeit einiger Gegner der Hinrichtung kam der Aussetzungsbefehl der Vollstreckung um wenige Stunden zu spät. Der deutsche Kaiser Wilhelm II. war über die Eigenmächtigkeit seines Brüsseler Statthalters verärgert und untersagte die Vollstreckung weiterer Todesurteile [1].

Es stellt sich die Frage, warum Benn 13 Jahre gewartet hatte, ehe er mit seinem Bericht an die Öffentlichkeit trat. Sicher greift die

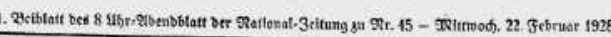
Mlbie Mití Cavell eridtosien wurde.

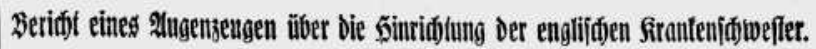
Voa Dr. Gottfried Benn, ehemaligem Oberart an Gouvenement Brissel.

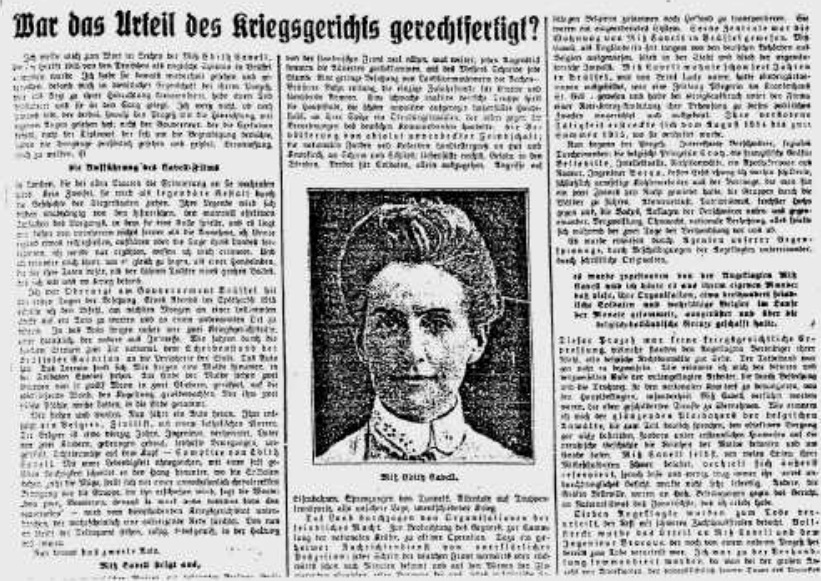

Abb. 2 Zeitungsartikel von Gottfried Benn über die Hinrichtung Edith Cavells (aus [3]).

Erklärung zu kurz, dass seine bekannte Abneigung gegen den Journalismus der Grund dafür war [2]. Im Bekanntenkreis Benns in Le Hulpe nahe Brüssel kursierte schon kurz nach der Hinrichtung Edith Cavells seine mündliche Schilderung des Ereignisses gegenüber Thea Sternheim, der Ehefrau des Dramatikers Carl Sternheim. Sie hatte am 3. Februar 1917 in ihrem Tagebuch festgehalten, wie entsetzt sie über die erschreckende Sachlichkeit Benns und seine Verteidigung der Korrektheit des Prozesses und der ordnungsgemäßen Exekution war [5]. Unmittelbar nach der Tötung Edith Cavells behauptete am 12. Oktober 1915 die „New York Times“, die Delinquentin habe die Schüsse des Hinrichtungspelotons überlebt und sei liegend vom kommandierenden Offizier durch einen sog. Fangschuss mit der Pistole getötet worden. Diese Version hielten offenbar beide kriegsführenden Parteien für so wahrscheinlich, dass von Seiten der Presseagenturen kein Interesse an einer Richtigstellung dieser Falschmeldung bestand [2].

Der eigentliche Anlass für das verzögerte Erscheinen von Benns Artikel war wahrscheinlich der 1928 in Großbritannien und Amerika gezeigte Spielfilm „Dawn“, in dem wiederum die britische Legende vom Überleben der Hinrichtung und der anschließenden Tötung Edith Cavells durch den Pistolenschuss dargestellt wurde. Es wurden Befürchtungen über mögliche Störungen des deutschamerikanischen Verhältnisses laut [2]. Edith Cavell wurde, nachdem sie zunächst in der Nähe der Hinrichtungsstätte beerdigt worden war, 1919 nach Großbritannien überführt und in ihrem Heimatort Norfolk beigesetzt ( Abb. 3). In England wurde sie zur Märtyrerin und Heldin, ihre Geschichte zum Gegenstand zahlreicher Bücher und Filme. Mit ihrem Porträt wurden Tausende Kriegsfreiwillige rekrutiert. In London und Melbourne wurden Statuen zu ihrem Andenken errichtet, in Kanada trägt ein Berg ihren Namen. In einer Sendung des ZDF-Magazins „Aspekte“ am 24. 9. 2004 wurde deutlich, dass das Schicksal von Edith Cavell in Großbritannien heute noch Anteilnahme erweckt. Die Beflissenheit, mit der Benn in seinem Artikel das Todesurteil gegen Edith Cavell rechtfertigte und ihren Fall von dem Justizmord an Sacco und Vanzetti in den USA abgrenzte [3], lässt aufhorchen. Als Gott- 


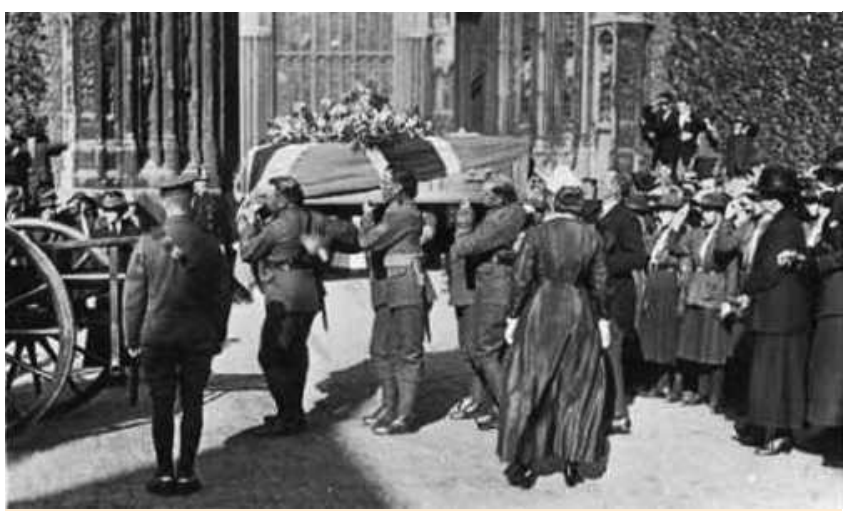

Abb. 3 Überführung des Sarges von Edith Cavell in ihren Heimatort Norfolk 1919 (Quelle: http://www.edithcavell.org.uk).

fried Benn Mitte der 30er-Jahre in der NS-Kampfschrift „Säuberung des Kunsttempels“ als „Kulturbolschewist“ bezeichnet wurde, führte er seinen Bericht über die Erschießung von Edith Cavell als Beweis seiner nationalen Gesinnung an [1].

Die kalte Sachlichkeit, mit der Benn die Exekution von Edith Cavell und Boog schilderte und kommentierte, wird auch damit erklärt, dass er den Vertretern der „Neuen Sachlichkeit“ zeigen wollte, dass er ihren literarischen Stil durchaus mühelos beherrschte [6]. Am wahrscheinlichsten ist wohl, dass es - wie auch andere Texte Benns zeigen - sein Bestreben war, Vorgänge mit dem sog. kalten Blick zu schildern, eine Art der distanzierten Darstellung von Vorgängen, wie sie von Nietzsche, Stendhal und Flaubert, später auch von Jünger und Brecht, kultiviert worden war $[1,2]$.

Benn versäumte nicht, die Tötung von Edith Cavell als Beleg für die Gnaden- und Sinnlosigkeit von Geschichte zu zitieren, die sich grausam über die Köpfe der Menschen hinweg vollzieht [3]. Max Rychner schrieb 1932 über Gottfried Benn: „Der Standort Benns ist unmenschlich, der eines kalten Gottes, der mit großen gefühllosen Augen die menschliche Tragikomödie anschaut, in die auch er verstrickt war, bevor er sich aus der Vergänglichkeit ins Zeitlose rettete“ [7]. Benn konnte sich in seinem Zeitungsartikel auch einen Seitenhieb auf die emanzipatorischen Bestrebungen der Frauen am Anfang des 20. Jahrhunderts nicht verkneifen: „Edith Cavell hat als Mann gehandelt und wurde von uns als Mann bestraft. Ich glaube, daß die Frau von heute für diese Konsequenz nicht nur Verständnis hat, sondern sie fordert" [3]. Gleichzeitig schleicht sich in Benns Text über die Hinrichtung Edith Cavells ein „öffiziöser Verlautbarungston“ [1] ein, wenn er schreibt: „Ich erinnere mich ihrer, um es gleich zu sagen, als einer Handelnden, die für ihre Taten büßte, als der kühnen Tochter eines großen Volkes, das sich mit uns im Krieg befand“ [1].

Was Thomas Mann-Verehrer einigermaßen überraschen dürfte, sind seine die Erschießung Edith Cavells betreffenden Sentenzen in den „Betrachtungen eines Unpolitischen“: „Was war es anderes als süßlicher Unernst und erbärmlicher Mangel an tragischem Sinn, wenn die Entente-Welt die standesrechtliche Erschießung einer englischen Frau beplärrte, die in Belgien ihr Pflegerinnenkleid mißbrauchte, um belgischen Soldaten über die Grenze zu helfen. [...] Man entehrte sie nicht, man ehrte sie, indem man sie vor die Flinten stellte“ [8]. Thomas Mann war im Gegensatz zu dem Augenzeugen Gottfried Benn der Meinung, Edith Cavell sei vom kommandierenden Offizier durch einen Pistolenschuss getötet worden, nachdem sie die eigentliche Erschießung überlebt hatte - ganz im Sinne der bereits genannten Legendenbildung. Hierzu heißt es dann bei Thomas Mann in ungewohnt hämischer und nationalistischer Weise: „Eine politische Handlung zu begehen, die vor die Flintenläufe führen kann, sollte nur der sich befugt und berufen glauben, der einigermaßen sicher ist, angesichts der Flintenläufe nicht ohnmächtig zu werden“ [8].

Johannes R. Becher und Egon Erwin Kisch griffen den Artikel Benns im „8 Uhr-Abendblatt“ heftig an, Kisch sprach vom Mord an der Krankenschwester Edith Cavell [2]. Es bleibt festzuhalten, dass Gottfried Benn seinen Bericht über die Hinrichtung Edith Cavells als emotionsloser Beobachter formuliert hat, der auch seine Funktion als Militärarzt bei der Feststellung des Todes der Delinquentin einschloss. Die in dem Artikel offenbarte Gefühlskälte des Arztes Dr. Gottfried Benn macht den Leser betroffen. Diesen Text hat er - wie auch andere kritikwürdige schriftliche und mündliche Äußerungen - später nicht verändert oder zurückgenommen. In diesem Zusammenhang ist erwähnenswert, dass Ernst Jünger seinen Bericht über die Erschießung eines deutschen Deserteurs 1941 in Frankreich in seinen Pariser Tagebüchern nachweislich zwei Mal retuschiert hat, sodass Jünger in der Endfassung nur als unbeteiligter Beobachter dasteht, obwohl seine früheren Tagebucheinträge zu diesem Vorgang Jüngers organisatorische Vorbereitungen an der Urteilsvollstreckung belegen [9]. Es ist ein schwacher Trost, dass man Gottfried Benn zumindest einen solchen Vorwurf der Retuschierung seines Berichtes nicht machen kann.

\section{Abstract}

\section{Unemotional Witness to a Tragedy \\ $\nabla$}

In October 1915, Dr. med. Gottfried Benn witnessed the trial of the nurse Edith Cavell in the German War tribunal in occupied Brussels. Edith Cavell was sentenced to death for leading troops to the enemy. As a military doctor he was ordered to the execution of the delinquent on the $12^{\text {th }}$ October 1915 . Only 13 years later he reported on this in an article for „8 Uhr-Abendblatt der Nationalzeitung“ - on the $22^{\text {nd }}$ February 1928. In this article Gottfried Benn describes the event, in an even for a doctor distant manner. The tragic fate of Edith Cavell has been subject of books, magazines and films. Up until now her fate has moved many British.

\section{Literatur}

1 Decker G. Gottfried Benn. Genie und Barbar. Biographie. Berlin: Aufbau, 2006

2 Lethen H. Der Sound der Väter. Gottfried Benn und seine Zeit. Berlin: Rowohlt, 2006

3 Benn G. Wie Miß Cavell erschossen wurde. Bericht eines Augenzeugen über die Hinrichtung der englischen Krankenschwester. 1. Beiblatt des 8 Uhr-Abendblatt der National-Zeitung zu Nr. 45 - Mittwoch, 22. Februar 1928, Berlin

4 Lenning W. Gottfried Benn mit Selbstzeugnissen und Bilddokumenten. Hamburg: Rowohlt, 1986

5 Ehrsam T. Gottfried Benn - Thea Sternheim. Briefwechsel und Aufzeichnungen. Göttingen: Wallstein, 2004

6 Rost N. Meine Begegnungen mit Gottfried Benn. In: Gottfried BennDen Traum alleine tragen. Neue Texte, Briefe, Dokumente. Wiesbaden: Limes, 1966

7 Rychner M. Nach dem Nihilismus. In: Hillebrand B (Hrsg). Über Gottfried Benn. Kritische Stimmen 1912 - 1956. Frankfurt/M.: S. Fischer, 1986

8 Mann T. Betrachtungen eines Unpolitischen. In: Thomas Mann. Gesammelte Werke. Bd. 12. Frankfurt/M.: S. Fischer, 1974

9 Krömer F. Vollstrecker und Betrachter. FAZ vom 16.6.2005 